\title{
AVES ANILHADAS NO BRASIL EM 1981 E RECUPERAÇÕES DE ANILHAS DESDE 1980
}

SUSANA DE MOURA LARA-RESENDE PAULO DE TARSO ZUQUIM ANTAS

Dando continuidade ao trabalho "Aves anilhadas no Brasil em 1980 e suas recuperações", apresentado por ocasião do IX Congresso Brasileiro de Zoologia, em Porto Alegre, RS, pretendemos apresentar aqui, dentro do mesmo estilo, as atividades de anilhamento no Brasil em 1981, e as recuperações de anilhas ocorridas desde o ano de 1980.

Salientamos uma vez mais o fato de que os dados de recuperação são de uso restrito dos anilhadores para fins de publicações científicas, sendo considerado aqui apenas a ocorrência ou não de recuperações.

$\mathrm{O}$ anilhamento no Brasil foi anteriormente descrito no que se refere à sua organização e uso (Antas, 1979; Antas \& Resende, 1982).

$\mathrm{Na}$ Tabela I estão listadas as aves anilhadas no Brasil de janeiro a dezembro de 1981, segundo os relatórios a nós remetidos pelos anilhadores. Cerca de 44 novas permissões de anilhamento foram emitidas ou renovadas em 1981, e um total de 2.549 aves foram anilhadas.

Os métodos mais utilizados foram o uso de redes de captura (mist-nets), armadilhas de telas de arame e anilhamento de filhotes em ninhais.

O número de aves anilhadas durante o ano de 1981 foi $53 \%$ mais baixo que em 1980, quando foram anilhadas 4.734 indivíduos. Essa diminuição foi motivada principalmente pela falta de anilhas, problema que só conseguimos solucionar no final do ano, quando recebemos nova remessa de anilhas através de doação da World Wildlife Fund - WWF. Não foi possível p. ex., dar continuidade no ano de 1981, ao anilhamento da pomba-de-bando Zenaida auriculata, iniciado em 1980 pelo convênio IBDF/Escola Superior de Agricultura de Mossoró - ESAM. O anilhamento de filhotes em ninhais, responsável por $70,41 \%$ do total de indivíduos anilhados em 1980 , foi bastante prejudicado pela falta de anilhas.

Em 1981, como em 1980, o uso de redes de captura nos diversos tipos e tamanhos, foi responsável pela grande diversidade de espécies anilhadas: 179 espécies pertencentes a 39 famílias tiveram representantes anilhados. A família Ardeidae, com 498 espécimes anilhados foi a mais representativa em termos de indivíduos, enquanto Tyrannidae teve o maior número de espécies com anilhas. A espécie Sula leucogaster, por sua vez, ocupou o lugar de espécie com maior número de indivíduos anilhados: 299, todos no estado de Santa Catarina.

Das aves anilhadas em 1980 e 1981, 58 tiveram suas recuperações relatadas ao Centro, o que perfaz uma taxa de recuperação de 2,27\% (Tabela II).

Considerando-se apenas os indivíduos anilhados em 1981 e recuperados no período de 1981-1982, teríamos uma taxa de recuperação de $0,78 \%$, ou seja, de 1,2 vezes maior que a taxa de $0,63 \%$ obtida no período de 1980-1981, para as aves anilhadas em 1980. Nota-se que, apesar da diminuição no número de aves anilhadas em 1981, a taxa de relatos de recuperação de anilhas foi maior, o que demonstra um interesse crescente por parte do público em geral, o qual, através da divulgação da técnica, tem aceitado e contribuído de maneira progressiva, aumentando a obtenção de dados das aves anilhadas.

Biólogos do Centro de Estudos de Migrações de Aves - CEMAVE. C.P. 04/034, Brasília, DF. 70312 . 
TABELA I: Aves anilhadas no Brasil em 1981, por espécie.

\begin{tabular}{lcc}
\hline NOME CIENTIFICO & NOME COMUM & $\begin{array}{c}\text { N. IND. } \\
\text { ANILHADOS }\end{array}$ \\
\hline
\end{tabular}

PODICIPEDIDAE

- Podilymbus podiceps

Mergulhão-Caçador

SULIDAE

- Sula leucogaster

Mergulhão

PHALACROCORACIDAE

- Phalacrocorax olivaceus Biguá

ANHINGIDAE

- Anhinga anhinga

Biguá-tinga

FREGATIDAE

- Fregata magnificensis

Fregata

200

ARDEIDAE

- Ardea cocoi

- Casmerodius albus

- Egretta thula

- Butorides striatus

- Nycticorax nycticorax

Garça-moura 76

Garça-branca-grande $\quad 161$

Garça-branca-pequena $\quad 169$

Socozinho 01

Savacu 91

THRESKIORNITHIDAE

- Phimosus infuscatus

Maçarico-de-cara-pelada

134

- Plegadis chihi

Caraúna

- Ajaia ajaja

Colhereiro

190

ANATIDAE

- Coscoroba coscoroba

Capororoca

Marreca pardinha $\quad 117$

- Anas flavirostris

- Anas georgica

Marreca-parda

Marreca-cricri

- Anas platalea

Marreca-colhereira

08

- Netta peposaca

Marrecão-da-patagônia

03

- Netta erythrophthalma

- Amazonetta brasiliensis

Marreca-pé-vermelho

01

- Cairinia moschata

- Oxyura dominica

Pato-do-mato

12

Marreca-bico-roxo

63

06

ACCIPITRIDAE

- Rosthramus sociabilis

Gavião-caramujeiro

ARAMIDAE

- Aramus guarauna

Carão

01

RALLIDAE

- Gallinula chloropus

Galinhola

- Fulica armillata

Carqueja-de-bico-maculado

- Fulica leucoptera

Carqueja-de-bico-amarelo

- Fulica rufifrons

Carqueja-de-bico-roxo

06 


\section{CHARADRIIDAE}

- Vanellus chilensis

- Pluvialis squatarola

- Charadrius semipalmatus

- Arenaria interpres

Quero-quero 13

Batuiruçu-de-axila-preta $\quad 02$

Batuira-norte-americana $\quad 09$

Vira-bosta 04

\section{SCOLOPACIDAE}

- Tringa solitaria

- Tringa flavipes

- Actitis macularia

- Calindris minutilla

- Calidris fuscicollis

- Calidris melanotos

- Calidris pusilla

- Numenius phaeopus

- Gallinago gallinago

Maçariquinho 01

Maçarico-de-perna-amarela $\quad 05$

Maçarico-pintado 04

Maçariquinho 05

Maçarico-de-sobre-branco $\quad 15$

Maçarico-de-colete $\quad 02$

Maçariquinho 08

Maçarico-de-bico-torto $\quad 01$

Narceja $\quad 01$

FURNARIIDAE

- Furnarius rufus

- Phleocryptes melanops

- Synallaxis ruficapilla

- Synallaxis spixi

- Synallaxis albescens

- Synallaxis cinerascens

- Certhiaxis cinamomea

- Poecilurus scutatus

João-de-barro 04

Bate-bico 04

Pichororé 03

João-teneném 03

Ui-pi 01

Pipui 02

Curutié 01

- Cranioleuca semicinerea

- Cranioleuca obsoleta

- Phacellodomus rufifrons

- Automolus leucophtalmus

- Xenops minutus

- Lochmias nematura

01

Arredio-oliváceo

06

02

Barranqueiro-de-olho-branco $\quad 04$

Bico-virado-liso 12

João-porca $\quad 01$

FORMICARIIDAE

- Thamnophilus caerulescens

Choca-da-mata $\quad 08$

- Thamnophilus torquatus

- Dysithamnus mentalis

- Myrmotherula unicolor

- Myrmotherula axillaris

- Pyriglena leuconota

- Pyriglena leucoptera

- Formicarius colma

- Conopophaga lineata

\section{PIPRIDAE}

- Pipra rubrocapilla

- Antilophia galeata

- Chiroxiphia pareola

- Manacus |manacus

- Machaeropterus regulus

- Neopelma pallescens

- Schiffornis virescens

- Schiffornis turdinus

Tangará-chifrudo $\quad 07$

Cabeça-encarnada $\quad 01$

Rendeira $\quad 37$

Choquinha-lisa $\quad 06$

Choquinha-cinzenta 01

Papa-formigas 01

Papa-taóca 01

Galinha-do-mato $\quad 01$

Chupa-dente 03

02

Flautim $\quad 01$ 
N. ${ }^{\circ}$ IND.

ANILHADOS

RYNCHOPIDAE

- Rynchops nigra

Talha-mar

COLUMBIDAE

- Columbina talpacoti

Rola-roca

- Columbina picui

Rolinha-picui

- Leptotila verreauxi

Juriti-pupu

Juriti-gemedeira

- Geotrygon montana

Pariri-vermelha

CUCULIDAE

- Crotophaga ani

Anu-preto

TYTONIDAE

- Tyto alba

Suindara

\section{ALCEDINIDAE}

- Ceryle torquata

Martim-pescador-grande

\section{BUCCONIDAE}

- Nystalus chacuru

João-bobo

PICIDAE

- Picumnus limae

- Picumnus cirratus

- Celeus flavescens

Pica-pau-anão-barrado

DENDROCOLAPTIDAE

- Dendrocynchla fuliginosa

- Sittasomus griseicapillus

Arapaçu-liso

Arapaçu-verde

- Lepdocolaptes angustirostris

Arapaçu-do-cerrado

- Lepdocolaptes fuscus

Arapaçu-rajado

TYRANNIDAE

- Colonia colonus

- Hymenops perspicillata

- Pyrocephalus rubinus

- Satrapa icterophrys

- Machetornis rixosus

- Muscivora tyrannus

- Tyrannus melancholichus

- Tyrannus albogularis

- Pitangus sulphuratus

- Myiarchus swainsoni

- Empidonax euleri

- Myiobus barbatus

- Myiophobus fasciatus

- Platyrinchus mystaceus

- Tolmomyas sulphurescens.

- Idioptilon mirandae

- Idioptilon orbitatum

- Capsiempis flaveola

- Pseodocolopteryx flaviventris

- Serpophaga subscritata

- Serpophaga nigricans

- Elaenia flavogaster

Viúva

Viuvinha-de-óculos $\quad 01$

Príncipe 02

Suiriri-pequeno $\quad 12$

Suiriri-cavaleiro 01

$\begin{array}{ll}\text { Tesourinha } & 02\end{array}$

Siriri 11

Siriri 01

Bem-te-vi 10

Irré 08

Enferrujado 13

Espoletinha 01

Felipe 11

Patinho 01

Bico-chato-de-orelha-preta 05

Tiririsinho-do-mato 07

Marianinha-amarela $\quad 01$

Amarelinho-do-junco $\quad 04$

Alegrinho 02

João-pobre 01

Guaracava-barriga-amarela $\quad 02$ 


\begin{tabular}{|c|c|c|}
\hline NOME CIENTIFICO & NOME COMUM & $\begin{array}{l}\text { N. IND. } \\
\text { ANILHADOS }\end{array}$ \\
\hline
\end{tabular}

- Elaenia parvirostris

- Elaenia cristata

- Elaenia chiriquensis

- Elaenia obscura

- Suiriri suiriri

- Sublegatus modestus

- Camptostoma obsoletum

- Santhomyas viresces

- Leptopogon amaurocephalus

- Pipromorpha oleoginea

- Pachiramphus viridis

- Pachiramphus polichpterus

HIRUNDINIDAE

- Phaeoprogne tapera

TROGLODYTYDAE

- Thryothorus leucotis

- Troglodytes aedon

MIMIDAE

- Mimus saturninus

TURDIDAE

- Turdus rufiventris

- Turdus leucomelas

- Turdus amaurochalinus

- Turdus fumigatus

- Turdus albicollis

SYLVIIDAE

- Polioptila dumicola

VIREONIDAE

- Cyclarhis gujanensis

ICTERIDAE

- Molothrus bonariensis

- Molothrus badius

PARULIDAE

- Parula pitiayumi

- Geothlypis aequinoctialis

- Basileuterus flaveolus

- Basileuterus culicivorus

- Basileuterus hypoleucus

- Basileuterus leucoblepharus

- Coereba flaveola

COEREBIDAE

- Dacnias cayana

THRAUPIDAE

- Euphonia violacea

- Tangara fastuosa

- Tangara cyanocephala

- Tangara cayana
Guaracava-de-bico-curto

15

Guaracava-de-crista 28

Tucão

Suiriri-cinzento

Guaracava-modesta

Risadinha

Piolhinho-verdoso

Cabeçudo

Supi

Taperá

Marido-é-dia

Cambaxirra

Sabiá-do-campo

01

Sabiá-laranjeira

Sabiá-barranco 23

Sabiá-branco $\quad 31$

Sabiá-da-mata 01

Sabiá-coleira 18

Balança-rabo-de-máscara

01

Gente-de-fora-vem

Vira-bosta

Asa-de-telha

Mariquita

01

Pia-cobra

Pula-pula

Pula-pula-assobiador

Sebinho

Saí-azul

Gaturamo-verdadeiro $\quad 06$

Pintor-verdadeiro 01

Saíra-lenço 05

Saíra-macaco 02 
N. ${ }^{\circ}$ IND.

ANILHADOS
- Thraupis sayaca

- Thraupis bonariensis

- Habia rubica

- Tachyphous rufus

- Tachyphonus coronatus

- Trichothraupis melanopis

- Hemithraupis flavicollis

- Thlypopsis sordida

- Neothraupis fasciata

- Schistochlamys melanopis

FRINGILLIDAE

- Saltator maximus

- Cyanoloxia glaucocaerulea

- Volatinia jacarina

- Tiaris fuliginosa

- Sporophila americana

- Sporophila collaris

- Sporophila lineola

- Sporophila nigricollis

- Sporophila caerulescens

- Sicalis flaveola

- Sicalis luteola

- Haplospiza unicolor

- Coryphospingus cucullatus

- Arremon flavirostris

- Zonotrichia capensis

- Poospiza nigrorufa

- Poospiza lateralis
Sanhaço-do-mamoeiro 08

Sanhaço-papa-laranja

11

Tié-do-mato-rosso

09

Pipira-preta

Sanhaço-frade

Tiê-de-topete

03

01

04

01

01

04

Bico-de-veludo

13

Trinca-ferro

01

Azulinho

01

Tiziu

09

Cigarra-coqueiro

01

Gola

02

Coleira-do-brejo

01

Bigodinho

03

Coleiro-baiano

13

Coleiro-papa-capim $\quad 19$

Canário-da-terra 11

Tipiu

02

Cigarra-bambu

03

Tico-tico-rei

03

Tico-tico-da-mata

Tico-tico

01

Quem-te-vestiu $\quad 02$

Quete

PLOCEIDAE

- Passer domesticus

Pardal

04

TOTAL

2.549

Conforme a fig. 02, as recuperações se deram em maior número entre as famílias de aves aquáticas e/ou cinegéticas, como era de se esperar. De acordo com o mapa 02 , as concentrações de recuperações são próximas a locais de anilhamento e/ou regiões de maior ocupação populacional. A recuperação de passeriformes torna-se mais difícil, uma vez que praticamente só ocorrerão quando tivermos um número maior de anilhadores utilizando redes de captura em habitats por eles freqüentados. As recuperações se deram principalmente devido à caça legal ou furtiva, e, em alguns casos, devido à ação de pescadores.

Embora nem todas as anilhas encontradas sejam relatadas ao Centro, temos notado que, após o início da divulgação do anilhamento através do folheto "UMA ANILHA DEVOLVIDA - O QUE ISTO REPRESENTA", bem como dos meios de comunicação e imprensa, um número cada vez maior de relatos tem chegado até nós. O crescente número de anilhamentos, e a continuidade nos trabalhos de divulgação permitirão uma maior participação do público, e a obtenção de nossos objetivos de conhecer a bioecologia da avifauna brasileira. 


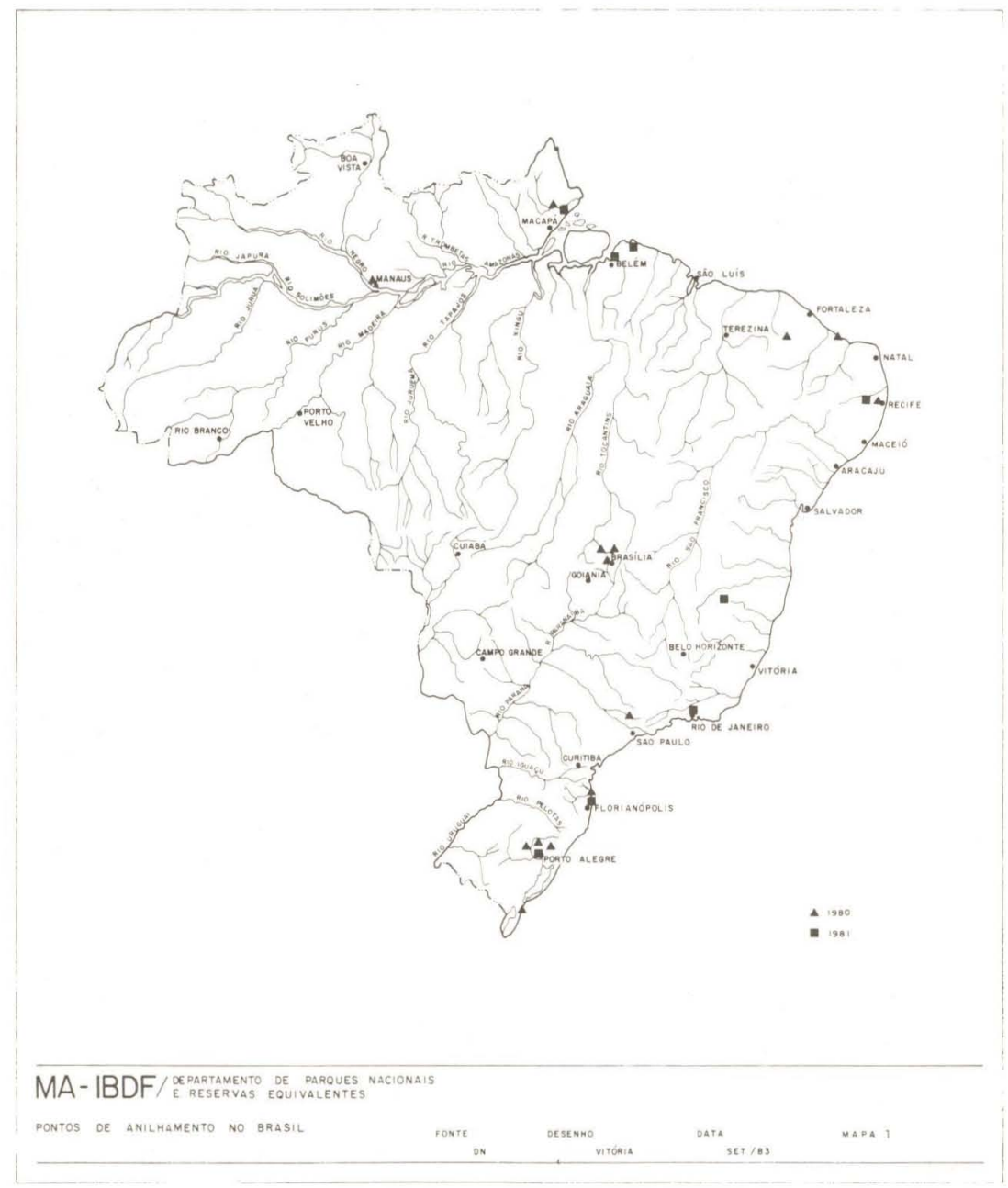




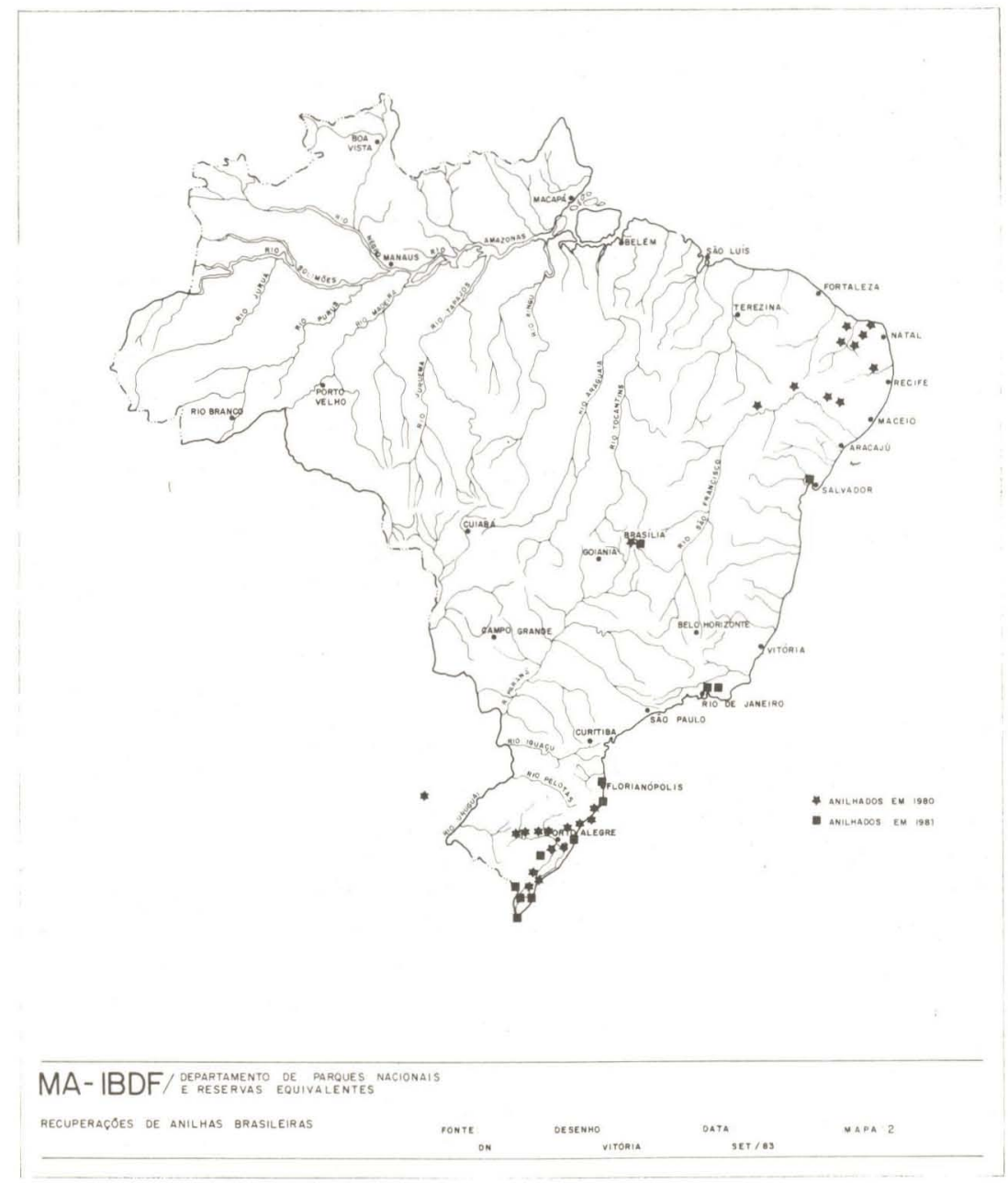


TABELA II: Aves recuperadas com anilhas do CEMAVE, anilhadas em 1980 e 1981.

$\begin{array}{lcc}\text { NOME CIENTIFICO NOME COMUM } & \text { N. DE } & \% \\ & \text { AVES } & \text { AVES } \\ \text { RECUP. } & \text { RECUP. }\end{array}$

\section{SULIDAE}

- Sula leucogaster

$06 \quad 2,0$

ARDEIDAE

- Casmerodius albus

Garça-branca-grande

- Egretta thula

Garça-branca-pequena

04

1,3

THRESKIORNITHIDAE

- Plegadis chihi

- Ajaia ajaja

Caraúna

Colhereiro

ANATIDAE

- Anas versicolor

- Amazonetta brasiliensis

- Dencrocygna viduata

RALLIDAE

- Gallinula chlorops

Marreca-cricri

Marreca-pé-vermelho

Irerê

Galinhola

01

2,2

Jaçanã

01

Maçarico-grande

01

12,5

- Tringa melanoleuca

COLUMBIDAE

- Zenaida auriculata

Pomba-de-bando

12

0,9

THRAUPIDAE

- Tachyphonus rufus

- Zonotrichia capensis

Pomba-de-bando

Tico-tico

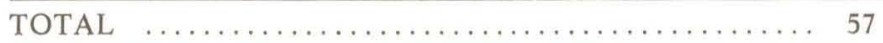

\section{AGRADECIMENTOS}

Agradecemos aos anilhadores por tornarem possível o presente trabalho através do envio dos relatórios de anilhamento em dia, e em especial aos recuperadores de anilhas, fornecendo os dados por nós solicitados, sem o que nossos esforços seriam em vão.

\section{REFERENCIAS}

Antas, P. T. Z., 1979. Present situation of bird banding in Brazil. The Ring 101: 96-98.

Leal, R. P.; P. T. Z. Antas; S. M. Lara Resende, 1981. Manual de Anilhamento de Aves, 106 pp. Brasil Florestal, M: A., Instituto Brasileiro de Desenvolvimento Florestal - IBDF, Brasília, DF. 